\title{
Microscopic Image Analysis of Nanoparticles by Edge Detection Using Ant Colony Optimization
}

\author{
Shwetabh Singh \\ Centre for Converging Technologies, University of Rajasthan, Jaipur-302004, India
}

\begin{abstract}
In this paper, I present an approach for analyzing nanoparticles microscopic images by edge detection using Ant Colony Optimization (ACO) algorithm to obtain a well-connected image edge map. Microscope image analysis of nanoparticles are subject to errors. Initially, the edge map of the image is obtained using various matlab toolbox conventional edge detectors \& adaptive thresholding. The end points obtained using such detectors are calculated. The ants are then placed at these points. The movement of the ants is guided by the local variation in the pixel intensity values. The probability factor of only undetected neighboring pixels is taken into consideration while moving an ant to the next probable edge pixel. The two stopping rules are implemented to prevent the movement of ants through the pixel already detected. The method is applied on the atomic force microscope (AFM) images of Cerium Oxide $\left(\mathrm{CeO}_{2}\right)$ nanoparticles \& SEM image of $\mathrm{ZnO}$ nanoparticles. The results show that the edges obtained in the images can be used for classification of particles, determining sizes \& shapes \& also distinguishing particles in agglomerates more precisely.
\end{abstract}

Keywords - Nanoparticles microscopic image, Ant Colony Optimization, Pheromone, Adaptive Thresholding

\section{Introduction}

Images of nanoparticles are obtained by various microscopic techniques like Atomic Force microscope(AFM), Scanning Electron microscope(SEM), Transmission Electron Microscope(TEM) etc. The characterization of particles obtained by these techniques is an important task. The main problem faced is distinguishing the particles in the agglomerates. Nanotechnology research and industrial products demanded strict nanoparticles characterization with a higher precision. The method of edge detection by Ant Colony Optimization in such images can be used to tackle above problems. The edge detectors are used to detect and localize the boundaries of objects in the images. An edge can be defined as sudden change of intensity in an image. In binary images, edge corresponds to sudden change in intensity level to 1 from 0 and vice versa. The Sobel operator is based on convolving the image with a small, separable, and integer valued filter in horizontal and vertical direction. The Prewitt [1] operator calculates the maximum response of a set of convolution kernels to find the local edge orientation for each pixel. The Canny detector [2] uses a multi-stage algorithm to detect a wide range of edges in images and defines edges as zero-crossing of second derivatives in the direction of greatest first derivative. Marr et al [3] proposed an algorithm that finds edges at the zero-crossings of the image Laplacian. Conventional edge detectors are usually performed by linear filtering operations. Non-linear filtering techniques for edge detection also saw much advancement through the SUSAN [4]. However, these methods often result in some drawback like the broken edges which leads to loss of information.

Many methods have been proposed in the past to link the broken edges too in order to improve the edge detection. Some edge linking approaches perform Hough transformation $[1,5]$ on edge image, then extract the specific shape to connect broken edges. However, the edges do not always have fixed shapes. Some other methods use hybrid techniques [6-7] to connect broken edges. Ant colony optimization (ACO) is heuristic method that imitates the behavior of real ants to solve the discrete optimization problem [25]. Ant colony optimization takes inspiration from the foraging behavior of some ant species [9]. A foraging ant deposits a chemical (pheromone) which increases the probability of following the same path by other ants. Ant colony optimization was formalized by Dorigo and co-workers.

The first ACO algorithm, called the ant system, was proposed by Dorigoetal[10]. Since then, a number of ACO algorithms have been developed, such as ant colony system [11], Max-Min ant system [12], ant colony algorithm for solving continuous optimization problem [13], an improved ACO for solving the complex combinatorial optimization problem [14-15]. Recently, a novel fuzzy ant system for edge detection [16], edge improvement by ant colony optimization[17], ant colony optimization and statistical estimation approach to image edge detection [18], adaptive artificial ant colonies for edge detection in digital images [19], are reported in the literature. The surface morphology of nanoparticles could be observed and characterized by TEM, SEM, AFM etc. And the nanoscale microscope images obtained by above instruments were usually characterized manually during the thirty years' development, which easily leaded to inefficient processing and errors[26]. In this study ant colony optimization is used to link the discontinuities in the edges in the microscopic images of nanopartricles while the edges are detected by adaptive thresholding. The edge point information supplied by 
the adaptive thresholding is more than that supplied by the Sobel operator. Therefore the study of applying ACO to the edges extracted from adaptive thresholding gives better results. The ACO methods are an iterative, probabilistic meta-heuristic for finding solutions to combinatorial optimization problems[8].

The rest of the study is organized as follows: Section 2 gives introduction of the of ant colony optimization. The whole technique for microscopic nanoparticles image edge detection is presented in Section 3. Section 4 presents experimental results \& final images and Section 5 gives conclusion.

\section{Ant Colony Optimization}

Ant colony optimization (ACO) is a nature-inspired optimization algorithm. It mimics the foraging behavior of ants. Ants deposit the chemical called as pheromone on the ground to mark paths to the food source. Pheromone is followed by other ants of the colony. Over the time, pheromone trails evaporates on the paths that are less followed by the ants and pheromone density increases on the shorter paths that are followed by more ants. The amount of pheromone evaporation depends on the time taken by the ants to travel down the path and back again.

In the ACO method, artificial ants use virtual pheromone to update their path through the image edges. It iteratively finds the optimal solution of the target pixels through the movements of ants over the image edges, by depositing and evaporating the pheromone. The probability for the ant's movement from one pixel to another is determined by probability transition matrix. During the $\mathrm{n}^{\text {th }}$ construction step, the $\mathrm{k}^{\text {th }}$ ant moves according to the probabilistic transition matrix defined by the Eq. (1) [22]:

$$
\operatorname{Pij}(n)=\frac{\left(\tau_{i j}{ }^{(n)}\right)^{\alpha}\left(\eta_{i j}\right)^{\beta}}{\sum_{j \in \Omega_{j}}\left(\tau_{i j}{ }^{(n)}\right)^{\alpha}\left(\eta_{i j}\right)^{\beta}}
$$

where $\tau_{\mathrm{i}, \mathrm{j}}^{(\mathrm{n})}$ is the pheromone information value, $\eta_{\mathrm{i}, \mathrm{j}}$ represents the heuristic information for pixel (x,y) for going from node $i$ to node $j$ which is calculated using Eq. (2), and the constants $\alpha$ and $\beta$ influence the pheromone information and heuristic information, respectively. There are eight possible neighboring pixels surrounding the central pixel at $(\mathrm{x}, \mathrm{y})$.

The $\eta_{\mathrm{i}, \mathrm{j}}$ is calculated as [15]

$$
\eta_{i j}=\frac{\max _{i j}\left(\begin{array}{l}
|I(x-1, y-1)-I(x+1, y+1)|, \\
|I(x-1, y+1)-I(x+1, y-1)|, \\
|I(x, y-1)-I(x, y+1)|, \\
|I(x-1, y)-I(x+1, y)|
\end{array}\right)}{\eta_{\max }}
$$

Where $\eta_{i j}$ is the heuristic information of pixel (x,y) and $\eta_{\max }$ is maximum heuristic value.

Pheromone intensity attracts the ant to follow the paths travelled by other ants. Hence, pheromone is updated twice, once for individual ant and secondly the global update. The $\tau_{\mathrm{i}, \mathrm{j}}{ }^{(\mathrm{n})}$ of individual ant is updated by[22]:

$$
\tau_{i j}^{(n)}=(1-\Psi) \cdot \tau_{i j}^{(n-1)}+\Psi \cdot \tau_{i j}^{(0)}
$$

where $\psi \epsilon[0,1]$ is the pheromone decay coefficient which diversifies the search by decreasing the desirability of edges that have already been traversed.

After the movement of all the ants, pheromone is updated globally using [16]:

$$
\tau^{(n)}=\left\{\begin{array}{l}
(1-\rho) \cdot \tau^{(n-1)}+\rho \cdot \Delta \tau^{(k)} \text { if }(i, j) \text { besttour }, \\
\tau^{(n-1)}, \text { otherwise }
\end{array}\right\},
$$

Where $\rho \epsilon[0,1]$ is the evaporation constant. $\Delta \tau^{(k)}$ is the amount of pheromone deposited by the ant which is given as follows[23]:

$$
\Delta \tau^{(k)}=\frac{C}{L^{k}}
$$

Where $\mathrm{L}^{\mathrm{k}}$ is the path length travelled by the $\mathrm{k}^{\text {th }}$ ant and $\mathrm{C}$ is a constant. 


\section{Whole Approach}

Extract the edges initially using matlab toolbox (3.1-3.6). The connectivity of the edges so obtained is then increased using ACO.

\subsection{Sobel Method}

The Sobel method finds edges using the Sobel approximation to the derivative. It returns edges at those points where the gradient of image is maximum.

\subsection{Prewitt Method}

The Prewitt method finds edges using the Prewitt approximation to the derivative. It returns edges at those points where the gradient of image is maximum.

\subsection{Canny Method}

The Canny method finds edges by looking for local maxima of the gradient of image. The gradient is calculated using the derivative of a Gaussian filter. The method uses two thresholds, to detect strong and weak edges, and includes the weak edges in the output only if they are connected to strong edges. This method is therefore less likely than the others to be fooled by noise, and more likely to detect true weak edges.

\subsection{Roberts Method}

The Roberts method finds edges using the Roberts approximation to the derivative. It returns edges at those points where the gradient of image is maximum.

\subsection{Laplacian of Gaussian Method}

The Laplacian of Gaussian method finds edges by looking for zero crossings after filtering image with a Laplacian of Gaussian filter.

\subsection{Zero-Cross Method} specify.

The zero-cross method finds edges by looking for zero crossings after filtering image with a filter you

\subsection{Edge Detection using Adaptive Thresholding}

Adaptive thresholding is used to separate desirable foreground image objects from the background based on the difference in pixel intensities of each region. Global thresholding uses a fixed threshold for all pixels in the image and therefore cannot deal with images containing a varying intensity gradient. Local adaptive thresholding, on the other hand, selects an individual threshold for each pixel based on the range of intensity values in its local neighbourhood. Adaptive thresholding takes a gray scale or color image as input and, outputs a binary image representing the edge information. For each pixel in the image, a threshold has to be calculated. If the pixel value is below the threshold it is set to the background value, otherwise it assumes the foreground value. The processed image is then used to obtain the end point information of the broken edges. The edges extracted from the above steps provide larger end point information as compared with that provided by above methods.

\subsection{Edge Improvement}

Discontinuities appear in the image after the application of any of the above methods. A central pixel position ( $\mathrm{x}, \mathrm{y}$ ) is considered as the endpoint if only one (out of eight) of the neighboring pixels is white and the central pixel itself is also white. This shown in Fig. 1, where the position $(x, y)$ is the end point. A number of ants is equal to the number of endpoints and they are placed at the positions of the endpoints.

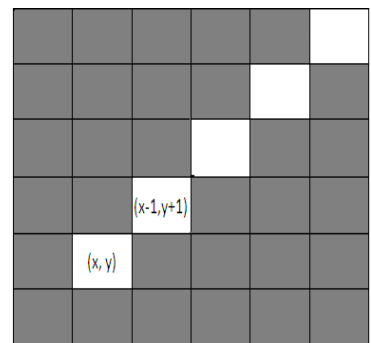

Fig.1 End point pixel (x,y) [16]. 
Transition rule: The transition rule takes into account the probability of undetected edge pixels only. For the ant movement, the probability factor for eight neighbouring pixels is calculated using probability transition matrix according to Eq. (1). The pixel with maximum probability factor is included in the set of edge pixels. To reduce the superfluous movement of ants the stopping rules proposed by [16] have been implemented.

Rule 1) The movement of the ant is stopped when it touches the track already traversed by another ant.

Rule 2) When all the neighboring pixels ( 8 pixels in $3 * 3$ grid) are already traversed by the ant, then the movement of ant stops.

\subsection{The Ant Colony Algorithm for Edge Improvement}

The ACO algorithm is used to increase the connectivity of the edges in the image obtained after applying conventional edge detection techniques. The steps are as follows:

1) Initialize the ant's position by placing them only at end points.

2) Initialise the pheromone matrix and calculate the heuristic information using Eq.(2)

3) Construction Process:

For the ant index $1: \mathrm{k}$

Move the $\mathrm{k}^{\text {th }}$ ant for $\mathrm{L}$ steps according to the probabilistic transition matrix using Eq. (1)

4) Calculate maximum probability of transition as per the transition rule and move the ant accordingly.

5) Perform local pheromone update process using Eq. (3)

6) Check whether all ants have moved one step, if yes, perform the global pheromone update using Eq. (4).

7) Check whether the ant can move to the next position by applying the stopping rules, if not, stop the ant.

8) Decision Process:

The pheromone matrix so produced is used to extract the complete edge trace by applying thresholding.

9) The edge pixels obtained are combined with the edge pixels obtained by adaptive thresholding to get the complete edge information.

\section{Results And Discussion}

This section presents the experimental results of the adaptive thresholding \& traditional edge detectors such as Canny, Prewitt, Sobel and they are improved by ACO. In these experiments, traditional edge detectors are executed by MATLAB toolbox. The codes for adaptive thresholding and ACO were also written in MATLAB. The results were obtained using the following values of parameters: $\alpha=0.5$ and $\beta=1$. The edge pixels are colored white on a black background.

Fig. 3(a)-(c) Shows the original images used for experiments of Cerium Oxide $\left(\mathrm{CeO}_{2}\right)$ nanoparticles from CCT, UoR \& (d) shows the SEM image of ZnO nanoparticles. The size of particles ranges from 200-600 $\mathrm{nm}$. The Fig. 4(a)-(d) shows the output after application of the above discussed technique of adaptive threshold \& ACO. It can be seen that the connected edge map is obtained, which can be used to distinguish particles in agglomerates and also determine the particle sizes \& shapes precisely. Fig. 5(a)-(d) shows results of Canny \& ACO. It shows some wrong edges which are noise \& double edges. Fig. 6(a)-(d) shows results of Prewitt \& ACO. It has many edge discontinuities. Fig. 7(a)-(d) shows results of Sobel \& ACO. This is comparable to adaptive threshold, but misses some edges. Adaptive Thresholding \& ACO gives the best results.

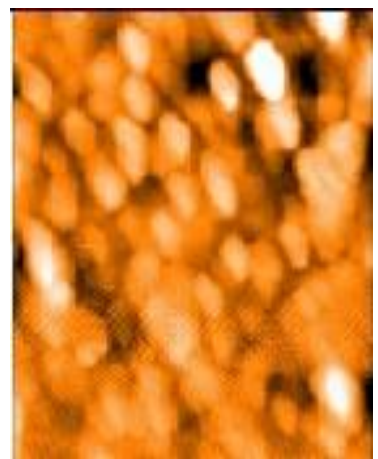

(a)

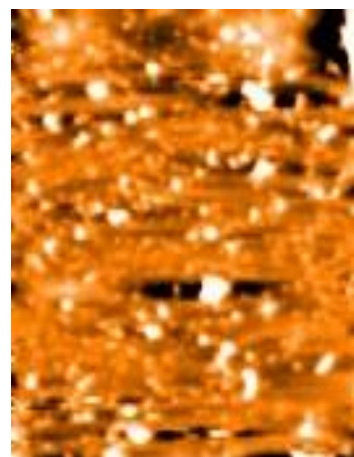

(b)

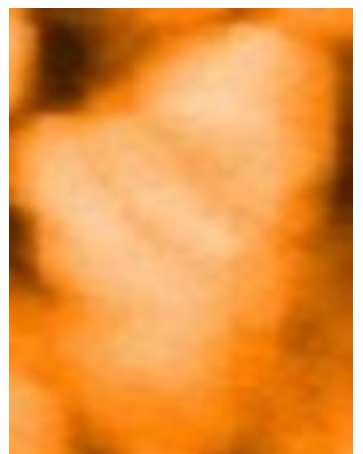

(c)

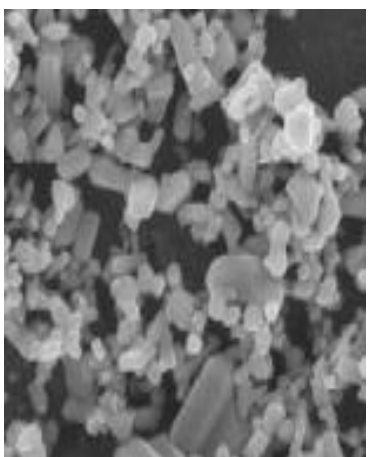

(d)

Fig.3 Original Images of Cerium Oxide $\left(\mathrm{CeO}_{2}\right)$ nanoparticles from AFM (a)-(c) \& SEM image of $\mathrm{ZnO}$ nanoparticles (d) 


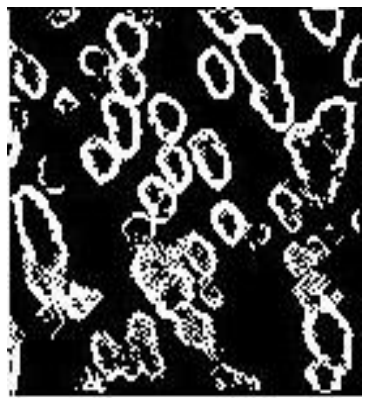

(a)

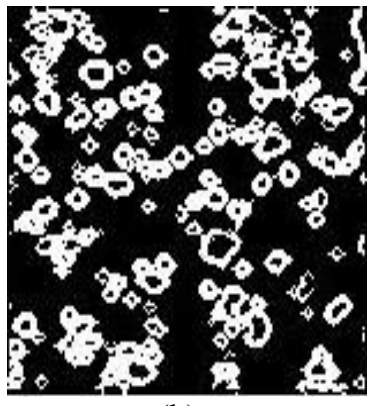

(b)

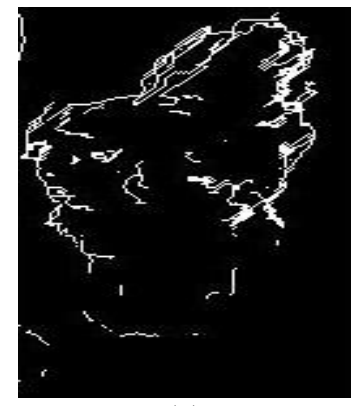

(c)

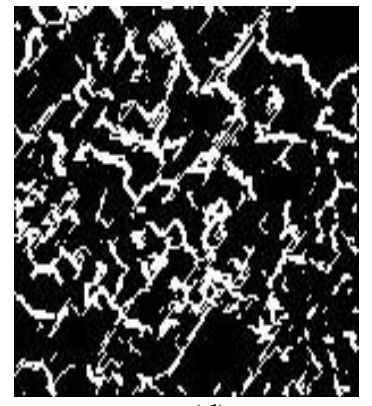

(d)

Fig.5 Final Edge Detected Images using Adaptive threshold \& ACO

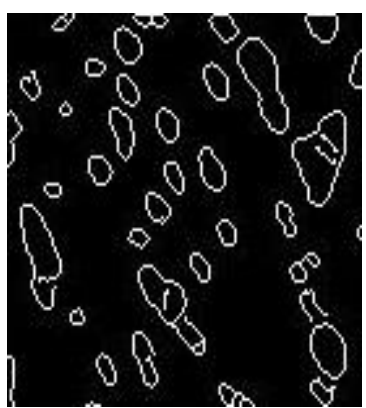

(a)

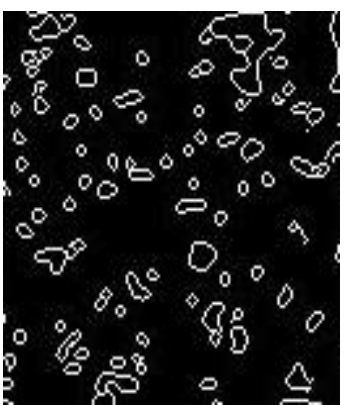

(b)

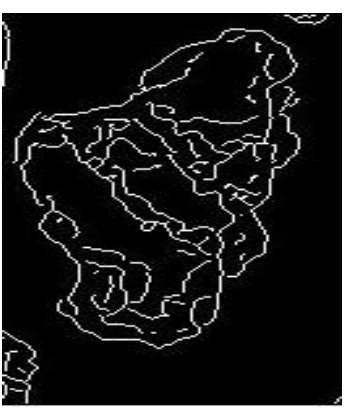

(c)

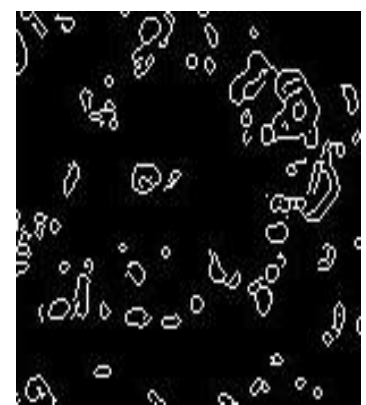

(d)

Fig.5 Final Edge Detected Images Canny \& ACO

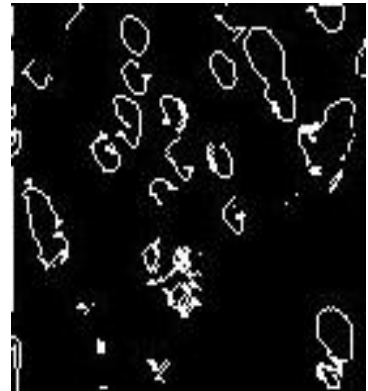

(a)

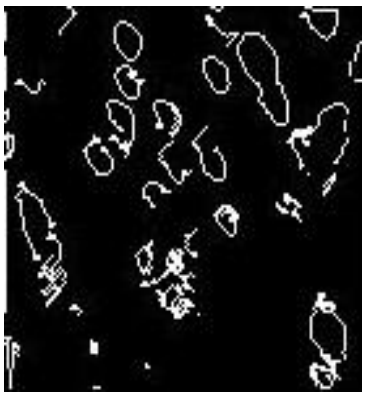

(a)

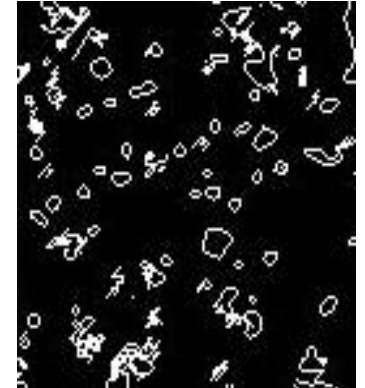

(b)

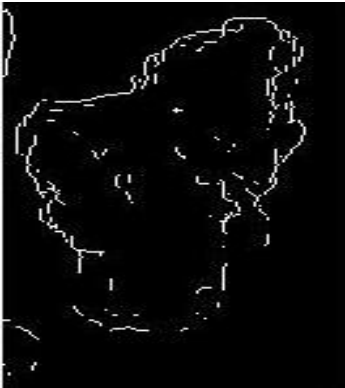

(c)

Fig.6 Final Edge Detected Images Prewitt \& ACO

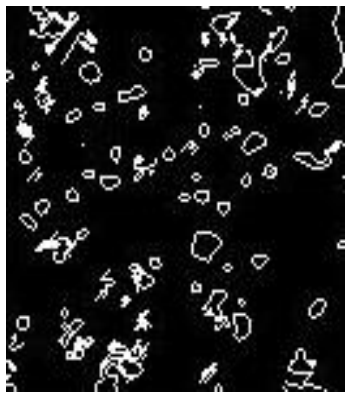

(b)

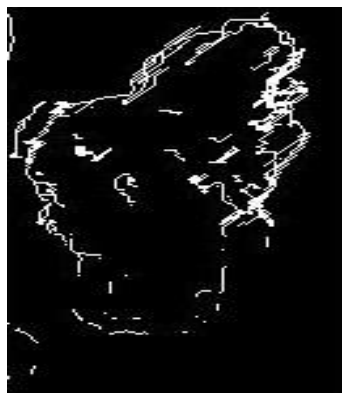

(c)

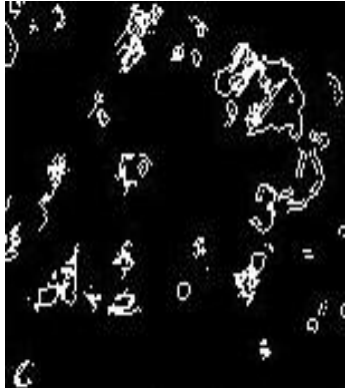

(d)

Fig.7 Final Edge Detected Images Sobel \& ACO

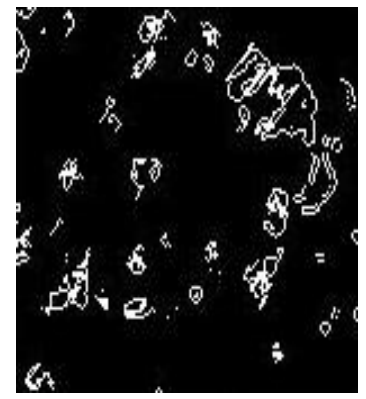

(d)

\section{Conclusion}

ACO along with other conventional edge detection techniques helps in improving \& analyzing the microscopic images of nanoparticles from various microscopic techniques such as AFM etc to a great extent. Adaptive threshold \& ACO gives the best results. The above method helps to distinguish particles in the agglomerates which are hard to visualize \& analyze. The sharp edges thus obtained by this approach helps in determining the sizes of the particles precisely. It also helps in determining the shapes of the particles. Particles can easily be distinguished from the substrate by this technique, thus making the image analysis much easy. This method is useful for removing the edge discontinuities in the images and can be used for image segmentation. 


\section{Acknowledgements}

I am extremely thankful to Prof. O.P. Verma, Delhi Technological University, New Delhi-India for his support and invaluable guidance for the completion of this work. I am also thankful to Prof. Ashok .K. Nagawat, University of Rajasthan, Jaipur-India.

Moreover I am thankful to my parents \& my younger brother for their faith in me and for being a source of encouragement. I am also thankful to my dear friends Sidharth Gupta and Tripti Singhal for their support.

\section{References}

[1] Gonzales, R.C., Woods, R.E., 2002. Digital Image Processing.Prentice Hall.

[2] Canny JF., "A computational approach to edge detection” IEEE Trans Pattern Anal Mach Intell1986;8(6):679-98.

[3] Marr, D., and Hildreth, E.C., "Theory of edge detection”,Proc. of the Royal Society of London,vol 207, $1980, p p$ 187-217.

[4] S.M.Smith and J.M.Brady ,'SUSAN- A new approach to low level image processing",Inetrnational Journal of Computer Vision23(1) 1997, pg 45-78.

[5] Parker, J.R., 1997. Algorithms for Image Processing and ComputerVision. Wiley Computer Publication.

[6] Ng, C.M., Leung, W.L., Lau, F. Edge detection using evolutionary algorithms. In: IEEE Internat. Conf. on System,Man and Cybernetics, Tokyo,Japan, 12th-15th October 1999, pp. 865-868.

[7] Sharifi, M., Fathy, M., Mahmoudi, M.T. A classified and comparative study of edge detection algorithms. In: IEEE Proc. Internat. Conf. on Information Technology: Coding and Computing, 8th-10th April 2002, pp. 117-120.

[8] Marco Dorigo, Thomas Stutzle "From Real to Artificial ants", MIT Press Book, ISBN0262042193, 2004.

[9] M. Dorigo, M. Birattari, and T. Stutzle, "Ant colony otimization," IEEE Computational Intelligence Magazine, vol. 1, pp. 28-39, Nov. 2006.

[10] M. Dorigo V. Maniezzo and A. Coloni,"Ant system: Optimization by a colony of cooperation agents," IEEE Trans on System Man and Cybernetics, Part B, Vol. 26, pp 29-41, Feb 1996.

[11] M. Dorigo and L.M. Gambardella,"Ant Colony System:" A cooperative learning approach to the travelling salesman problem." IEEE Trans. On Evolutionary Computation, Vol 1,Number 1, pp.53-61.April 1997.

[12] T. Stutzle and H. Holger H, "Max-Min ant system”, Future Generation Computer Systems, vol. 16, pp. 889-914, Jun. 2000.

[13] Li Hong, ZiongShibo, “ On Ant Colony Algorithm for solving Continuous Optimization Problem” IEEE International Conference on Intelligent Information Hiding and Multimedia Signal Processing, Harbin USA,15-17 August 2008, pp 1450-1453.

[14] Jingan Yang, YanbinZhuang,"An Improved ant colony optimization algorithm for solving a complex combinatorial optimization problem "Applied Soft Computing, Volume 10 ,Issue 2: March 2010.

[15] HosseinNezamabadi-pour, SaeidSaryazdi, EsmatRashedi "Edge detection using ant algorithms" Soft Computing,2006,Number 6,Vol 10: Pg:623-628.

[16] O.P. Verma, M. Hanmandlu, Ashish Kumar Sultania, Dhruv "A Novel Fuzzy Ant System for Edge Detection", Computer and Information Science (ICIS), 2010 IEEE/ACIS 9th International Conference, Yamgata Japan, 18th-20th August 2010 , Page(s): 228 233.

[17] Das. Lu, C.C. Chen, "Edge Detection improvement by ant colony optimization “, Pattern Recognition Letters Vol 29, Issue 4, 2008, pp 416-425

[18] Jian Zhang, Kun He, Jiliu Zhou, Mei Gong “Ant Colony Optimization and Statistical Estimation Approach to Image Edge Detection"WiCOM, Chengdu,China, 23th-25th September 2010 ,IEEE, Page(s): 1 - 4.

[19] Jevtić, A., Andina, D. “Adaptive Artificial Ant Colonies for Edge Detection in Digital Images"IECON,Glendale, AZ, USA, 7th-10th November 2010, IEEE, Page(s): 2813 - 2816

[20] O.P. Verma, M. Hanmandlu, P. Kumar, and A. Jindal “A Novel Bacterial Foraging Technique for Edge Detection", Pattern Recognition Letters Vol. 32, pp. 1187-1196, 2011.

[21] N. Otsu "A Threshold Selection method from gray level histograms," IEEE Trans.Syst.,Man,Cybern,Vol 9,pp 62-66,Jan 1979.

[22] O.P. Verma, M. Hanmandlu, Punit Kumar, ShivangiShrivastava "A novel approach for edge detection using ant colony optimization and fuzzy derivative technique",Advance Computing Conference, 2009.IEEE international, Patiala India, 6th-7th March 2009 page(s):1206 - 1212

[23] Sergey Subbotin and Alexey Oleynik "Modifications of Ant Colony Optimization Method for Feature Selection " CADSM'2007, 20th-24th February, 2007, Polyana, UKRAINE.

[24] Xiang Ming, Wu XiaopeiHuaQuanping "A Fast Thinning Algorithm for Fingerprint Image" The 1st International Conference on Information Science and Engineering (ICISE2009),Nanjing, China,18th-20th December 2009.

[25] Dorigo, M., St\}utzle, T., 2004. Ant Colony Optimization. MIT Press.

[26] Bohua Feng, Siyuan Mo, 2012. The authors - Published by Atlantis Press, Morphological Characteristics of Drug-loade Nanoparticles based on Microscope Images Analysis System. 